\title{
UMA ABORDAGEM PROBABILÍSTICA PARA A AVALIAÇÃO DE RISCOS EM CADEIAS DE SUPRIMENTO
}

\section{A PROBABILISTIC APPROACH TO ACCESS RISK IN SUPPLY CHAINS}

\author{
Kevin McCormack* kmccorm241@aol.com \\ Marcelo Bronzo** marcelobronzo@cepead.face.ufmg.br \\ Marcos Paulo Valadares de Oliveira** mpvo@ufmg.br \\ *Universidade do Estado da Carolina do Norte - NCSU/ \\ ${ }^{* *}$ Universidade Federal de Minas Gerais \\ ${ }^{* * *}$ Universidade Federal do Espírito Santo
}

\begin{abstract}
Resumo: Este artigo apresenta uma proposta envolvendo a aplicação do conceito de redes Bayesianas para o gerenciamento de riscos em cadeias de suprimento, especificamente no relacionamento de díades fornecedor-cliente, a partir do mapeamento de categorias de risco e de eventos correlatos. $\mathrm{O}$ trabalho pode ser identificado como uma tentativa de contribuição à construção de modelos preditivos que permitam dar suporte ao processo de tomada de decisão gerencial, no tocante ao gerenciamento e mitigação de riscos. No artigo, um exemplo hipotético foi desenvolvido para se ilustrar a potencialidade de aplicação de redes Bayesianas para os fins propostos. $O$ artigo detalha, por fim, a potencialidade do modelo teórico-conceitual proposto, bem como de questões relevantes para futuras pesquisas sobre o tema.
\end{abstract}

Palavras-chave: Gerenciamento de risco; Redes bayesianas, Rupturas de estoques; Efeito chicote; Cadeias de suprimento.

Abstract: This paper presents a proposal involving the application of the concept of Bayesian networks for risk management in supply chains, specifically on the relationship of supplier-customer dyads from the mapping of risk categories and related events. The work can be identified as an attempt to contribute to the construction of predictive models that allows supporting the process of decision making with regard to managing and mitigating risks. In the article, a hypothetical example was designed to illustrate the potential application of Bayesian networks for their purposes. The article details, finally, the potential of the theoretical-conceptual model proposed, as well as relevant issues for future research on the topic.

Key-words: Risk management; Bayesian networks; Inventory disruptions; Bullwhip effect; Supply chains.

\section{INTRODUÇÃO}

Em um esforço para alcançarem vantagem competitiva por meio da redução de custos e melhoria na responsividade de suas operações, as organizações procuram assegurar maior foco em suas competências centrais e a desverticalização de partes de suas atividades produtivas e de inovação. A intensificação das práticas 
de terceirização pode representar o aumento da dependência de uma organização em relação aos seus fornecedores e, consequentemente, é uma decisão que poderá modificar o seu perfil de risco ${ }^{1}$. Nesse sentido, as organizações são instadas crescentemente a desenvolverem capacidades para avaliação de riscos de rupturas em seus fluxos com fornecedores diretos e, em sentido amplo, nos processos em cadeias de suprimentos (LOCKAMY III; MCCORMACK, 2010).

O gerenciamento do risco de rupturas é uma temática relevante para as empresas que buscam melhorar a coordenação e o balanceamento de seus recursos internos e externos, de forma a se tornarem mais responsivas e eficientes, reduzindo lead times e minimizando estoques (em princípio, há um trade-off claro aqui). É preciso, para tanto, estimar os níveis de risco aceitáveis associados às possíveis rupturas e, então, desenvolver estratégias e diretrizes para o gerenciamento de tais riscos.

O problema torna-se mais complexo, naturalmente, ao se focalizar o fenômeno da internacionalização das cadeias de suprimentos. Ao considerar o movimento das empresas para a adoção de uma perspectiva global em suas fontes de fornecimento, é inevitável que neste contexto possam ser observadas novas oportunidades, mas igualmente novos desafios. Se por um lado fontes globais de fornecimento proporcionam preços reduzidos de compra e acesso cada vez mais amplo aos mercados, por outro, a complexidade operacional de um canal de distribuição global aumenta não somente a probabilidade de risco de rupturas no fluxo de produtos e serviços no longo fluxo da cadeia de suprimentos, mas também a própria magnitude de tais rupturas, e de seus efeitos (ELKINS et al., 2005).

Este artigo, de natureza teórica e de caráter exploratório, tem como objetivo discutir o tema de gerenciamento de riscos em cadeias de suprimento, destacandose a aplicação do modelo probabilístico de redes bayesianas como um recurso útil a esta finalidade. A partir de um estudo bibliográfico e de esforços analíticos visando integrar conceitos relevantes à discussão, este trabalho intenciona contribuir para a

\footnotetext{
${ }^{1}$ Os perfis de risco são compostos de eventos de risco associados à rede de suprimentos, às operações internas ou aos fatores externos à empresa (LOCKAMY III; MCCORMACK, 2010).
} 
orientação de futuras pesquisas sobre o assunto, ainda pouco explorado no campo da Gestão de Operações.

Após esta breve seção de introdução, vem apresentada na seção 2 a compilação de estudos teórico-conceituais relacionados ao gerenciamento de cadeias de suprimentos, gestão de estoques e gerenciamento de riscos em cadeias de suprimentos. Na seção 3, vem apresentada a conceituação de redes bayesianas, bem como o desenvolvimento de um exemplo de sua aplicação tratando do risco de ruptura de estoques em processos logísticos com fornecedores. Ainda na seção 3, vem apresentado um modelo hipotético-conceitual contemplando variáveis para a mensuração da probabilidade de risco de rupturas de estoques em cadeias de suprimentos, bem como algumas questões relevantes, atinentes aos construtos do modelo, para subsidiar futuros estudos empíricos sobre o tema. Por fim, na seção 4 do trabalho, são apresentadas as considerações finais do artigo.

\section{GESTÃO DA CADEIA DE SUPRIMENTOS}

O risco de rupturas causadas tanto por fatores internos às cadeias de suprimentos - definidas como redes de organizações envolvidas em diferentes processos e atividades que produzem valor na forma de produtos e serviços entregues ao consumidor final (MENTZER et al., 2001; CHRISTOPHER, 1997) quanto por forças do ambiente externo é, atualmente, um assunto importante tanto nos meios empresariais quanto acadêmicos. As cadeias de suprimento tem sido objeto de estudos para o desenvolvimento de abordagens que visam a identificação, avaliação, análise, e tratamento de áreas de vulnerabilidade e riscos em cadeias de suprimentos (NEIGER et al., 2009).

A complexidade inerente aos fluxos de transações nos mercados internacionais, seja entre empresas ou com consumidores finais, afeta as características do projeto das redes logísticas crescentemente, fazendo uso de fornecedores globais para inúmeros bens e serviços. Essa globalização das fontes de suprimento estimula a busca por maneiras mais eficazes de coordenar o fluxo de materiais nos processos da empresa. Um fator crítico para a efetividade dessa coordenação é o relacionamento integrado com os fornecedores da cadeia. Há 
indícios crescentes na literatura técnica indicando que o processo de monitoramento e compartilhamento de informações na cadeia de suprimentos, através de soluções tecnológicas compartilhadas entre os agentes, contribui para o efetivo ganho no gerenciamento e mitigação de riscos envolvidos nos processos de suprimento (PECK, 2006).

Há nas atividades de projeto e de planejamento dos processos em cadeias de suprimentos, quase sempre, uma orientação das empresas para um melhor posicionamento no espectro custo vs. responsividade, quando são considerados os atributos de desempenho mais valorizados pelos seus clientes. Uma vez que as decisões gerenciais que objetivam 0 aumento da responsividade para um determinado sistema logístico inevitavelmente envolvem custos adicionais para o sistema, tornam-se rapidamente compreensíveis as razões pelas quais as empresas, em suas atividades de planejamento, procuram definir uma solução em termos de eficiência (de custos) que seja adequada a um determinado nível de responsividade. Entre outras variáveis, este nível de responsividade, que está intrinsecamente relacionado às práticas de gestão de estoques, será então definido em função de características da demanda, e por atributos valorizados por cada segmento de clientes a ser atendido pela empresa.

\subsection{Gestão de estoques e problemas de coordenação de fluxos em cadeias de suprimentos}

A condição para uma melhor administração dos estoques ao longo de cadeias de suprimento está em tornar maior a visibilidade do fluxo de recursos e de informações que percorrem toda a cadeia - quanto mais reconhecidos e monitorados forem esses fluxos, menor será a necessidade de estoques de segurança para atender à demanda e às necessidades de produção. Em linhas gerais, isso significa que quanto menos informação sobre produto e mercado possui a empresa, ou quanto mais restritos mostram-se os seus fluxos de informação com outras empresas, maior será a necessidade de elementos de precaução (estoques, 
fundamentalmente) para atender às flutuações na demanda, o que traz impactos diretos nas margens de lucros da firma.

Como as cadeias de suprimento mais convencionais compreendem entidades empresariais com o mínimo de transparência em relação às informações relacionadas ao mercado, é inevitável que essas cadeias procurem usar estoques para se precaverem das oscilações e incertezas da demanda.

Um dos principais problemas encontrados nas decisões de suprimento é a variabilidade entre a demanda prevista e a demanda real. A variabilidade nos padrões de emissão de pedidos e na demanda de uma cadeia muitas vezes é ocasionada pela modificação dos pedidos, contrariando a previsão de demanda e ocasionando mudanças significativas em termos de quantidade e mix de produtos. Os efeitos negativos dessas mudanças se propagam na cadeia de suprimentos, especialmente para os agentes situados à montante na rede.

Por conseguinte, essas cadeias são forçadas a manterem seus estoques dimensionados muito além do necessário, duplicando-o a cada interface comprador/fornecedor. Tal fato não acarreta apenas uma sobrecarga significativa em termos de capital de giro, mas fundamentalmente, faz com que essas cadeias tornem-se lentas em responder à demanda volátil, sobretudo do ponto de vista da flexibilidade qualitativa.

Um problema adicional das cadeias de abastecimento com elevados níveis de estoques posicionados entre clientes e fornecedores é que as pequenas mudanças na demanda do mercado final aumentam e se distorcem na medida em que se movem para trás na cadeia. Quanto mais distante estiver o elo da fonte da variabilidade, maiores serão os efeitos negativos decorrentes das mudanças na previsão de demanda. Tal efeito, reconhecido como efeito chicote, é gerador de um considerável custo oculto para a cadeia de abastecimento como um todo. $O$ efeito chicote, também conhecido como efeito Forrester (FORRESTER, 1961), tem suas raízes no fato de que, em uma cadeia com vários membros, com cada um agindo independentemente do outro e compartilhando apenas o mínimo de informações, é muito provável que até pequenas alterações na demanda do usuário final resultem em uma ampliação exacerbada da demanda (à montante). 
As causas desse fenômeno de "onda gigantesca" ou "chicotada" assentam-se principalmente no fato de que estoques independentes a cada fase da cadeia agem como reservas que distorcem as exigências e, na verdade, ocultam dos fornecedores a demanda real. Um estoque superdimensionado em um atacadista ou varejista, por exemplo, oculta a demanda real para um fornecedor à montante do processo, pelo menos até o momento de um novo pedido de ressuprimento do atacadista ou varejista. $O$ resultado é uma instabilidade severa nos programas de produção nas empresas à montante da rede, afetando negativamente as eficiências globais da cadeia, com empresas tendo de programar horas extras quando 0 "chicote" oscila para cima e pagar caros períodos de ociosidade quando o "chicote" oscila para baixo. Isso implica custos crescentes que, no final, serão pagos pelo único elo que alimenta a rede de valores monetários - o usuário final. Em outras palavras, estas ineficiências somam-se, ao longo da cadeia de suprimentos, para contribuir com aumentos no preço do produto ao cliente final.

Uma das fontes de distorção da demanda observada pelos diferentes elos da cadeia vem do fato que, muito provavelmente, cada um dos elos enxerga apenas sua demanda imediata, que vem distorcida pelas políticas de estoques dos seus clientes, dos clientes dos seus clientes, e assim por diante. Como as políticas de estocagem dos clientes, clientes dos clientes, e assim por diante, são independentemente estabelecidas (já que não há coordenação da rede), as distorções crescem em amplitude quanto mais à montante da rede estiverem localizadas essas empresas.

A ausência de um fluxo contínuo de informações de mercado entre os vários elos da cadeia, além de demandas distorcidas, pode gerar indisponibilidade de produtos causada pela incapacidade de um fornecedor de atender um pedido diferente daquele que era previsto. Uma falha de suprimento em determinado elo pode afetar todos os elos subseqüentes, caso as empresas não tenham agilidade e flexibilidade para enfrentar uma ruptura em seus estoques. Não apenas distorções nas demandas, mas também problemas na consistência dos prazos de entrega de mercadorias ou problemas de qualidade (não-conformidade) dos produtos, ou até mesmo situações de exceção como greves de funcionários ou catástrofes naturais, 
dentre outros elementos, são aspectos que representam riscos em maior ou menor grau e, conseqüentemente, exercem impactos no abastecimento de toda a cadeia.

A avaliação e o gerenciamento de riscos em cadeias de suprimento podem permitir, até certo ponto, a redução tanto da ocorrência quanto dos efeitos negativos das rupturas de estoques para as empresas. Neste sentido, o gerenciamento de risco minimiza a probabilidade de rupturas diante de eventos imprevistos, proporcionando uma maior continuidade no abastecimento, com o mínimo de falhas (ZSIDISIN et al., 2004).

\subsection{Gestão de riscos em cadeias de suprimentos}

Em qualquer tipo de relacionamento em cadeias de suprimentos há algum nível de risco ou incerteza. De acordo com Elkins et al. (2005), a gestão de risco em cadeias de suprimentos é uma tarefa desafiadora, pois o risco deriva de uma variedade de múltiplas fontes, tais como incêndios, atrasos no transporte, redução na velocidade do trabalho ou mesmo a interrupção forçada por greves e desastres naturais.

Atualmente, com a tendência das empresas operarem de forma enxuta e não possuírem grandes estoques ou excesso de capacidade, a possibilidade de que ocorram perdas de produção causadas por falta dos recursos necessários aumenta constantemente. Como resultado, problemas no fluxo de materiais podem rapidamente gerar conseqüências em toda a rede de suprimentos.

As abordagens para gestão de riscos em cadeias de suprimentos têm procurado mensurar tanto atributos dos fornecedores quanto das estruturas de suprimento com o objetivo de comparar fornecedores e prever rupturas. Os resultados são então utilizados para preparar devidas ações para a mitigação dos riscos e estratégias de resposta associadas com tais fornecedores (LOCKAMY III; MCCORMACK, 2010). A gestão de risco em cadeias de suprimentos envolve a identificação dos danos/perdas potenciais, o entendimento da probabilidade de potenciais danos e uma associação de criticidade para tais danos (GIUNIPERO; ELTANTAWY, 2004). 
Neste sentido, a rede de suprimentos é inerentemente vulnerável a rupturas e falhas em qualquer um dos elementos envolvidos em suas operações (RICE e CANIATO, 2003; BLACKHUST et al., 2005). Enquanto para muitas empresas ainda não foi possível quantificar o custo de rupturas ou de crises em suas cadeias de suprimentos, algumas empresas têm apresentado alguns avanços nesta área. Como exemplo, Rice e Caniato (2003) apresentaram resultados de uma pesquisa que estimou um impacto de $\bigcup \$ 50$ a $\bigcup \$ 100$ milhões nos custos diretos de uma empresa para cada dia que a sua rede de suprimentos permanece em ruptura.

Zsidisin (2003) define risco de suprimento como uma possibilidade de ocorrência de um incidente associado ao abastecimento interno a partir de uma ruptura individual de um fornecedor ou do mercado de suprimento como um todo, o que resulta na incapacidade da empresa em atender as demandas dos clientes ou em ameaças à vida e à integridade física e segurança de seus clientes. Segundo Handfield e McCormack (2007), existem seis categorias de risco dividas entre características intrínsecas do fornecedor e fatores situacionais. A cada categoria estão associados eventos que resultaram na ruptura de estoques daquele fornecedor, o que gera impactos negativos para o mesmo e para seus clientes. A Figura 1 apresenta a classificação proposta por Handfield e McCormack (2007), abordando desde as categorias de risco até os efeitos da ruptura de estoque do fornecedor. 


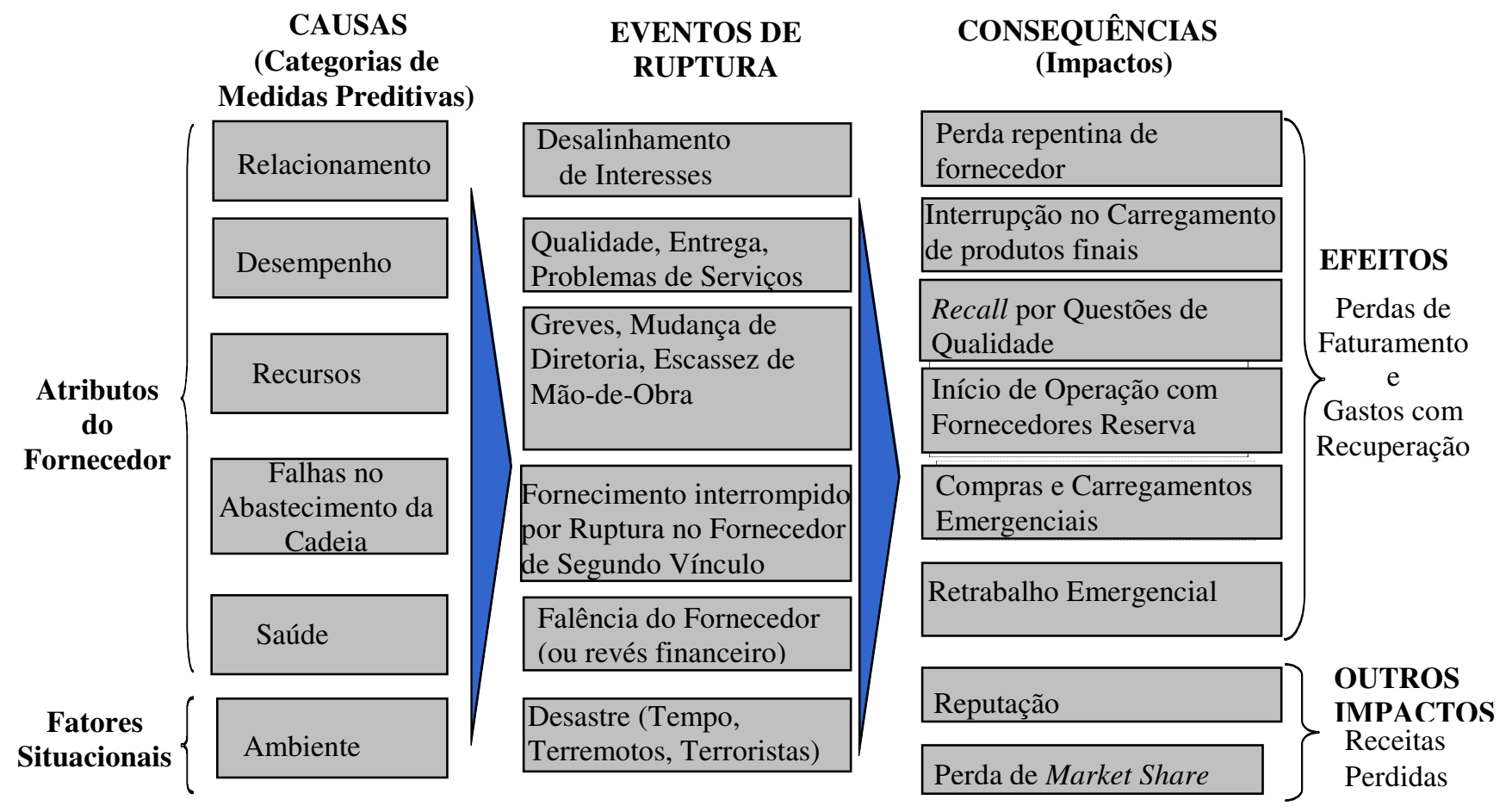

Figura 1: Estruturação das Relações de Risco de Suprimento Fonte: Handfield e McCormack (2007)

Os fatores de risco não são mutuamente exclusivos, sendo que a ocorrência de um fator pode implicar na ocorrência de outro. Se determinado fornecedor não pode ampliar suas economias de escala ou de escopo no curto prazo para atender uma demanda crescente ou exigências de flexibilidade no mix de produtos, ele poderá ser um elemento restritivo localizado no sistema. Mas também se este mesmo fornecedor não puder se adequar às mudanças tecnológicas no longo prazo, ele também provavelmente não será capaz de produzir e atender os volumes exigidos por essa demanda crescente no futuro (ZSIDIN, 2003).

Ao compreender bem as fontes, bem como a classificação dos riscos de suprimento, torna-se possível estabelecer estratégias que permitam minimizá-los de maneira eficiente. Isto é, quando se detecta que a ruptura de estoque ocorreu por um atraso de entrega do fornecedor dado sua restrição de capacidade para atender a todo o pedido no tempo desejado, pode-se trocar de fornecedor, dividir o pedido entre mais fornecedores ou mesmo adotar outra solução que pareça ser mais adequada ao novo contexto. 


\section{REDES BAYESIANAS}

Os modelos determinísticos de gestão de estoques como, por exemplo, o Lote Econômico de Compra (LEC), muitas vezes assumem premissas que não condizem com o contexto real em que as decisões de estoque devem ser tomadas. Assume-se, por exemplo, que a demanda é determinística e constante ao longo do tempo, e que seu fator constante é calculado por uma projeção simples de vendas com base em eventos passados. Valores históricos, entretanto, terão maior valor intrínseco se as condições fundamentais do problema permanecerem as mesmas em um período futuro, para o qual se fazem as projeções.

Como as empresas operam em mercados altamente competitivos, dados históricos podem não se apresentar como uma fonte suficiente para definir as estratégias de estoque. As Redes Bayesianas, por outro lado, permitem a consideração de variáveis intervenientes nas estratégias sob a luz das condições presentes de negócio, sem o apoio exclusivo de eventos passados (PHILIPS; DAWSON, 1968). O modelo bayesiano define previamente probabilidades aos níveis de estoque, permitindo a incorporação de eventos futuros no processo de decisão (PHILIPS; DAWSON, 1968).

As Redes Bayesianas constituem um modelo gráfico que representa as relações probabilísticas entre as variáveis de um sistema. Tais redes são sempre representadas por um conjunto de nós e um conjunto de arcos. Cada nó representa uma determinada variável aleatória e cada variável deve ter um número finito de estados mutuamente exclusivos, como Verdadeiro e Falso. Cada arco representa uma relação causal entre as variáveis, sendo que o arco é direcionado da causa para o efeito com o símbolo de uma seta (FENTON; NEIL, 2007; HAMMOND; O'BRIEN, 2001). A cada nó, pode-se associar uma tabela de probabilidade denominada Tabela de Probabilidades do Nó (TPN).

Considerando-se o evento "Greve de caminhoneiros" como um evento de probabilidade marginal (ou incondicional), as probabilidades de ocorrência de greve poderiam estar representadas como indicado na TPN (Tabela 1), abaixo: 
Tabela 1: TPN para Greve de Caminhoneiros

$$
\begin{array}{lll}
\text { Greve de } & \text { Falso } & 0,95 \\
\cline { 2 - 3 } \text { Caminhoneiros } & \text { Verdadeiro } & 0,05
\end{array}
$$

Na Figura 2, a seguir, podemos assumir, portanto, que todos os nós são discretos, com dois estados possíveis, "Verdadeiro" ou "Falso" (no mundo real, um nó como "Ruptura Fornecedor 2" poderia assumir uma escala contínua, representando o número de unidades faltantes). Os arcos representam as relações de influência ou causais entre as variáveis. Assim, uma greve de caminhoneiros poderia causar e ou influenciar tanto uma ruptura no fornecedor 1 quanto no fornecedor 2; enquanto chuvas e inundações poderiam, também, causar ou influenciar a ruptura no fornecedor 1 .

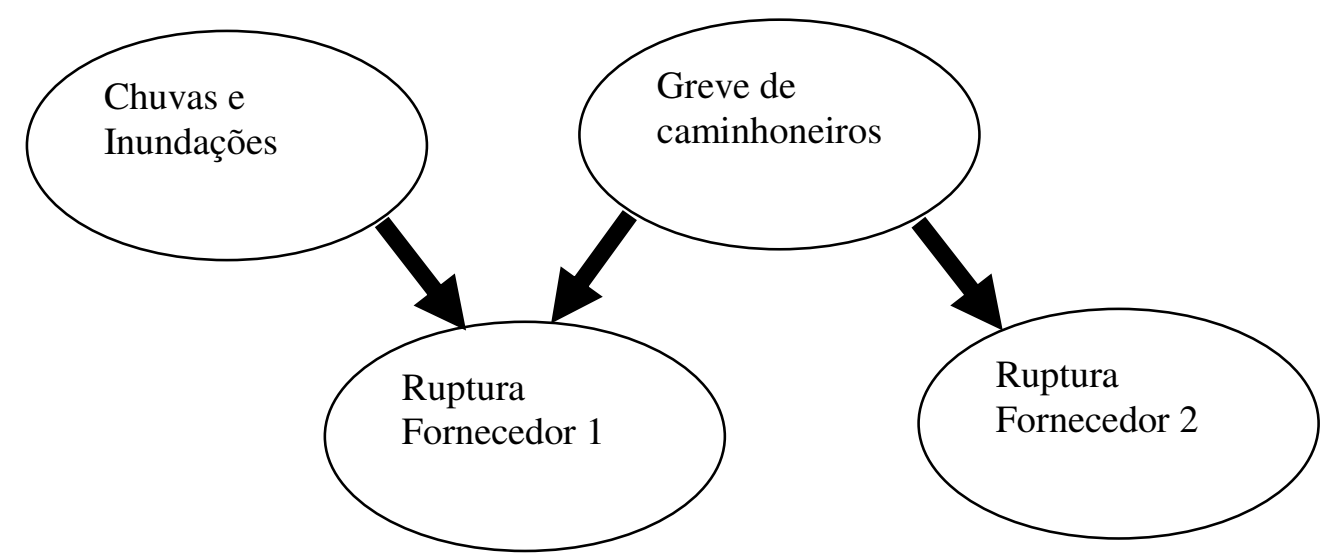

Figura 2: Rede bayesiana simples

A característica-chave das redes bayesianas é que elas nos habilitam a modelar e a refletir sobre incertezas. Neste exemplo, uma greve de caminhoneiros não implica necessariamente em uma ruptura no fornecedor 2 , mas há um aumento na probabilidade que esta ruptura possa ocorrer. Tal informação é capturada na TPN da "Ruptura Fornecedor 2" como ilustrado na Tabela 2, abaixo. A TPN para cada nó mapeia a probabilidade condicional de cada possível resultado, considerando-se a combinação de resultados dos nós anteriores. Neste caso, o nó "Ruptura Fornecedor 2" tem um nó anterior, "Greve de caminhoneiros". 
Tabela 2: TPN para Ruptura Fornecedor 2

Greve de Caminhoneiros

\begin{tabular}{llcc} 
& & \multicolumn{2}{c}{ Caminhoneiros } \\
\hline Ruptura & & Falso & Verdadeiro \\
\cline { 2 - 4 } Fornecedor & Falso & 0,9 & 0,2 \\
\cline { 2 - 4 } 2 & Verdadeiro & 0,1 & 0,8
\end{tabular}

Assim, a TPN nos diz que:

- A probabilidade de haver ruptura no fornecedor 2 , dado que há greve de caminhoneiros, é de 0,8;

- A probabilidade de não haver ruptura no fornecedor 2, dado que há greve de caminhoneiros, é de 0,2;

- A probabilidade de haver ruptura no fornecedor 2, dado que não há greve de caminhoneiros, é de 0,1 ;

- A probabilidade de não haver ruptura no fornecedor 2, dado que não há greve de caminhoneiros, é de 0,9 .

Usualmente, existem várias formas para se determinar as probabilidades nas TPN's. Para a greve de caminhoneiros, podem ser utilizados dados de quantos eventos desse tipo ocorreram no passado para se estimar a probabilidade de sua ocorrência futura. Alternativamente, caso dados estatísticos não estejam disponíveis, podem ser utilizadas probabilidades subjetivas de acordo com as opiniões de especialistas. A característica-chave das redes bayesianas, portanto, é a de que podem ser acomodadas tanto as probabilidades baseadas em dados subjetivos quanto em dados objetivos, ou uma combinação de ambas.

Após construir as TPN's podem ser então utilizadas as probabilidades bayesianas para executar diversos tipos de análises. A probabilidade bayesiana é portanto, fundamentalmente, uma revisão das probabilidades à luz das observações atuais dos eventos (FENTON e NEIL, 2007). Suponha que uma ruptura no fornecedor 2 seja identificada. Então, intuitivamente, com base na rede de 
probabilidades anteriormente identificada, a probabilidade de ocorrência de uma greve de caminhoneiros deve ter aumentado o seu valor anterior de 0,05. Mas qual a magnitude desse aumento? O teorema de Bayes proporciona a resposta para esta questão, conforme ilustrado no Quadro 1, abaixo. Como identificado no referido Quadro, a crença revisada é de que a probabilidade de ter havido uma greve de caminhoneiros considerando que foi observada uma ruptura do fornecedor 2 , será de 0,296, e não mais 0,05 (como indicado na Tabela 1, anteriormente apresentada). Com base em tal informação, podem ser também revisadas as crenças acerca da ruptura do fornecedor 1 . Assim, a probabilidade anterior aumenta desde que se saiba que existe uma ruptura do fornecedor 2. Portanto, torna-se possível identificar o aumento da probabilidade de ruptura no fornecedor 1 a partir da identificação de uma ruptura no fornecedor 2 , e vice versa. Na prática, tais cálculos são feitos automaticamente em qualquer ferramenta de redes bayesianas, tais como Agenarisk, Bayesware Discoverer, Netica e Microsoft MSBNx Bayesian Network Editor and Tool Kit (FENTON; NEIL, 2007).

Suponha que G represente "Greve de Caminhoneiros" e RF2 represente "Ruptura Fornecedor 2". Iniciamos com uma probabilidade de G anterior que chamaremos de $P(G)$, mas estaremos interessados em conhecer qual é a probabilidade de $G$ posterior dada a evidência de RF2. Escrevemos isso como $P(G / R F 2)$. O teorema de Thomas Bayes é representado pela seguinte fórmula utilizada para calcular $P(G / R F 2)$ :

$$
P(G / R F 2)=\frac{P(R F 2 / G) P(G)}{P(R F 2)}
$$

Sabemos, a partir da TPN, que $P(R F 2 / G)=0,8$ e que $P(G)=0,05$. Então, o numerador no teorema de Bayes é 0,04 . O denominador, $\mathrm{P}(\mathrm{RF} 2)$, é considerado como probabilidade marginal (ou incondicional) que haja uma ruptura no fornecedor 2 - é a probabilidade que haja ruptura no fornecedor 2 sem que não haja nenhuma informação específica sobre os eventos (neste caso a greve de caminhoneiros) que influenciam RF2. A TPN não proporciona este valor diretamente, mas ela proporciona indiretamente por meio da equação:

$$
\begin{aligned}
& \mathrm{P}(R F 2)=\mathrm{P}(R F 2 / G) \mathrm{P}(\mathrm{G})+\mathrm{P}(R F 2 / \text { not } \mathrm{G}) \mathrm{P}(\text { not } \mathrm{G}) \\
& =0.8(0.05)+0.1(0.95) \\
& =0.135
\end{aligned}
$$

Então, substituindo este valor de $\mathrm{P}(\mathrm{RF} 2)$ no teorema de Bayes temos que $\mathrm{P}(\mathrm{G} / \mathrm{RF} 2)=$ $0.04 / 0.135=0.296$.

Quadro 1: Teorema de Bayes aplicado ao problema de ruptura em fornecedores 
Quando identificadas evidências de rupturas nas redes de suprimento e sendo essas informações utilizadas para atualizar as demais probabilidades da rede, chamamos isso de Propagação (FENTON e NEIL, 2007). Na teoria, pode-se inserir qualquer observação/evidência em qualquer lugar na rede bayesiana e utilizar-se do conceito de propagação para atualizar as probabilidades marginais de todas as variáveis não observadas/identificadas. Tal procedimento pode alavancar análises poderosas que não seriam possíveis a partir do uso de outros métodos estatísticos.

\subsection{Aplicações da modelagem bayesiana no gerenciamento de riscos em cadeias de suprimentos}

Pai e colaboradores (2003) foram, provavelmente, os primeiros pesquisadores a avaliarem os riscos de cadeias de suprimentos utilizando redes bayesianas. $O$ estudo examinou o perfil de risco associado com uma cadeia de suprimentos de explosivos (TNT) do Departamento de Defesa norte americano (DoD). A cadeia de suprimentos era composta de fábricas, pontos de armazenagem e depósitos locais. Utilizando redes bayesianas, os pesquisadores foram aptos a estabelecer fatores de riscos e limites aceitáveis de riscos para todos ativos pertencentes à cadeia de suprimentos do DoD.

A modelagem bayesiana permite a medição de forma sistemática da ocorrência de fatores de risco que possam levar a eventos de baixa freqüência e alta severidade. Como conseqüência, tais modelos são utilizados para medir o risco operacional, identificar a influência dos fatores de risco, calcular a sensibilidade nos eventos de perda e simular a distribuição de perdas e cenários de perda excessiva (MARQUES; DUTRA).

Os modelos probabilísticos apresentam-se como alternativas para contornar os problemas normalmente encontrados na medição de riscos operacionais, uma vez que é comum a insuficiência de dados e, normalmente quando existem, são dados históricos. As Redes Bayesianas podem ser utilizadas para tomar decisões baseadas em probabilidades, decidir quais evidências adicionais devem ser observadas a fim de se obter informações úteis do sistema e analisar o sistema a fim 
de buscar os aspectos do modelo que possuam maior impacto sob as variáveis de consulta (MARQUES; DUTRA).

Em relação as outros modelos probabilísticos disponíveis, as Redes Bayesianas apresentam vantagens por serem facilmente compreendidas, dado que as relações entre as variáveis são, em grande parte, intuitivas. Outra vantagem é que este modelo provê informações sobre o efeito de possíveis intervenções nas variáveis da rede, bem como demanda menor tempo computacional de solução, uma vez que normalmente os algoritmos de Redes Bayesianas são menos complexos que outros modelos probabilísticos.

Contudo, as Redes Bayesianas se fundamentam em distribuições de probabilidade que, embora não sejam conhecidas a priori, não mudam ao longo do tempo para as variáveis contempladas em um modelo. Outra consideração está relacionada ao nível de confiança de um item. Embora a taxa de ruptura seja desconhecida por ser inicialmente indeterminada, ela não varia significativamente ao longo do tempo, e será cada vez mais exata quando os dados reais de ruptura forem fornecidos (AZOURY; MILLER, 1984).

Quando se trata da gestão de estoques, a incerteza sobre a ocorrência de rupturas é inerente ao processo de gestão de estoque, dado que existem inúmeras variáveis externas e internas que podem afetar o nível de estoque. Isto é, nas decisões de estoque há risco operacional envolvido. A ruptura de estoque em determinado elo repercute em toda a cadeia de suprimentos, uma vez que há variabilidade na entrega de matérias-prima e produtos (AZOURY; MILLER, 1984). As Redes Bayesianas, portanto, podem ser utilizadas como modelos preditivos para o gerenciamento de ruptura de estoques em cadeias de suprimento.

\subsection{Modelo de Handfield e McCormack (2007) para gerenciamento de riscos em cadeias de suprimento}

O modelo conceitual apresentado neste artigo tem com base os trabalhos de Handfield e McCormack (2007) sobre Gerenciamento de Risco de Suprimento. Tais autores objetivam a identificação de variáveis direcionadas para a mensuração do risco de ruptura de estoques por parte dos fornecedores. De acordo com Handfield e 
McCormack (2007), existem seis categorias de risco para associados a um fornecedor:

- Relacionamento

- Desempenho

- Recursos Humanos

- Falhas no Abastecimento da Cadeia

- Saúde Financeira

- Ambiente

Para cada categoria de risco, existem diferentes eventos que podem ser tomados como causas de rupturas no estoque de um fornecedor, o que, por sua vez, pode gerar rupturas de agentes diretos ou indiretos vinculados a este fornecedor.

Com o objetivo de avaliar a probabilidade de ruptura em cada evento e, conseqüentemente identificar a probabilidade de ruptura de cada fornecedor, ou seja, o risco de ruptura gerado pelo fornecedor em uma cadeia de suprimentos, utilizou-se como referência o trabalho de Handfield e McCormack (2007) para identificar os 25 indicadores críticos representados pelos seus respectivos índices de probabilidade $\mathrm{P}(\mathrm{E} 1)$ a $\mathrm{P}(\mathrm{E} 25)$, conforme detalhado na Tabela 3, a seguir. 


\begin{tabular}{|c|c|c|c|}
\hline & $\begin{array}{l}\text { Categorias de } \\
\text { Risco }\end{array}$ & Eventos & Probabilidade \\
\hline \multirow{25}{*}{ 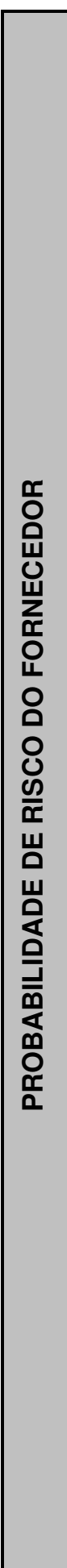 } & \multirow{4}{*}{ Relacionamento } & $\begin{array}{l}\text { Falta de compartilhamento de dados entre cliente } \\
\text { e fornecedor }\end{array}$ & $P(E 1)$ \\
\hline & & Inexperiência do fornecedor & $P(E 2)$ \\
\hline & & O cliente não é crítico para o fornecedor & $P(E 3)$ \\
\hline & & Interesses conflitantes entre cliente e fornecedor & $\mathrm{P}(\mathrm{E} 4)$ \\
\hline & \multirow{6}{*}{ Desempenho } & $\begin{array}{l}\text { Controle ineficaz da qualidade dos processos e } \\
\text { dos produtos }\end{array}$ & $P(E 5)$ \\
\hline & & Baixa responsividade no suporte técnico & $P(E 6)$ \\
\hline & & Incapacidade de melhoria contínua & $P(E 7)$ \\
\hline & & Falta de flexibilidade para lidar com incidentes & $\mathrm{P}(\mathrm{E} 8)$ \\
\hline & & Inabilidade de responder a variações na demanda & $P(E 9)$ \\
\hline & & Falhas ou atrasos na entrega & $P(E 10)$ \\
\hline & \multirow{3}{*}{$\begin{array}{l}\text { Recursos } \\
\text { Humanos }\end{array}$} & Erros ou faltas da força de trabalho & $\mathrm{P}(\mathrm{E} 11)$ \\
\hline & & Rompimento de sociedade & $\mathrm{P}(\mathrm{E} 12)$ \\
\hline & & Greve sindical & $P(E 13)$ \\
\hline & \multirow{5}{*}{$\begin{array}{c}\text { Falhas no } \\
\text { Abastecimento } \\
\text { da Cadeia }\end{array}$} & $\begin{array}{l}\text { Falhas na entrega do fornecedor de segunda } \\
\text { ordem }\end{array}$ & $P(E 14)$ \\
\hline & & Mudanças no processo produtivo & $P(E 15)$ \\
\hline & & Falta de comunicação entre cliente e fornecedor & $P(E 16)$ \\
\hline & & Única fonte de suprimento & $\mathrm{P}(\mathrm{E} 17)$ \\
\hline & & Imprecisão dos dados & $P(E 18)$ \\
\hline & \multirow{2}{*}{$\begin{array}{c}\text { Saúde } \\
\text { Financeira }\end{array}$} & Revés financeiro ou falência do fornecedor & $\mathrm{P}(\mathrm{E} 19)$ \\
\hline & & $\begin{array}{l}\text { Falta de compartilhamento de dados financeiros } \\
\text { entre cliente e fornecedor }\end{array}$ & $P(E 20)$ \\
\hline & \multirow{5}{*}{ Ambiente } & $\begin{array}{l}\text { Incapacidade de acompanhar a dinâmica do } \\
\text { mercado }\end{array}$ & $P(E 21)$ \\
\hline & & $\begin{array}{l}\text { Reestruturação dos processos causada por } \\
\text { fusões ou aquisições }\end{array}$ & $P(E 22)$ \\
\hline & & Mudanças nas regulamentações & $\mathrm{P}(\mathrm{E} 23)$ \\
\hline & & Falhas no transporte & $P(E 24)$ \\
\hline & & Desastres políticos ou ambientais & $P(E 25)$ \\
\hline
\end{tabular}

Quadro 2: Eventos de risco classificados por categorias

Fonte: Handfield e McCormack (2007)

Com a técnica de redes bayesianas, pode-se calcular a probabilidade de ruptura do fornecedor a partir das probabilidades de ocorrência dos eventos. Isto é, Revista Produção Online, v.10, n.3, p. 577-598, set., 2010 
determina-se o risco do fornecedor pela probabilidade de ruptura do mesmo, dado que um ou mais eventos ocorreram. A mensuração das probabilidades dos 25 indicadores, portanto, é a base para o cálculo de risco de cada fornecedor da rede.

De modo análogo, pode-se determinar a probabilidade de ruptura não de um único agente, mas de toda a rede de suprimentos. Nesse caso, os indicadores são os vários fornecedores que compõem a rede e o evento é a ruptura em determinado fornecedor. Como as probabilidades de risco de cada fornecedor já estarão calculadas, pode-se calcular a probabilidade de ruptura da rede, dado que houve falha no suprimento de um ou mais fornecedores da cadeia.

Agrupando cada evento e suas respectivas categorias, torna-se possível idealizar um modelo hipotético que identifique o risco de ruptura da cadeia de suprimentos com base no risco de ruptura de cada fornecedor. As dimensões do modelo estão graficamente representadas na Figura 3, abaixo.

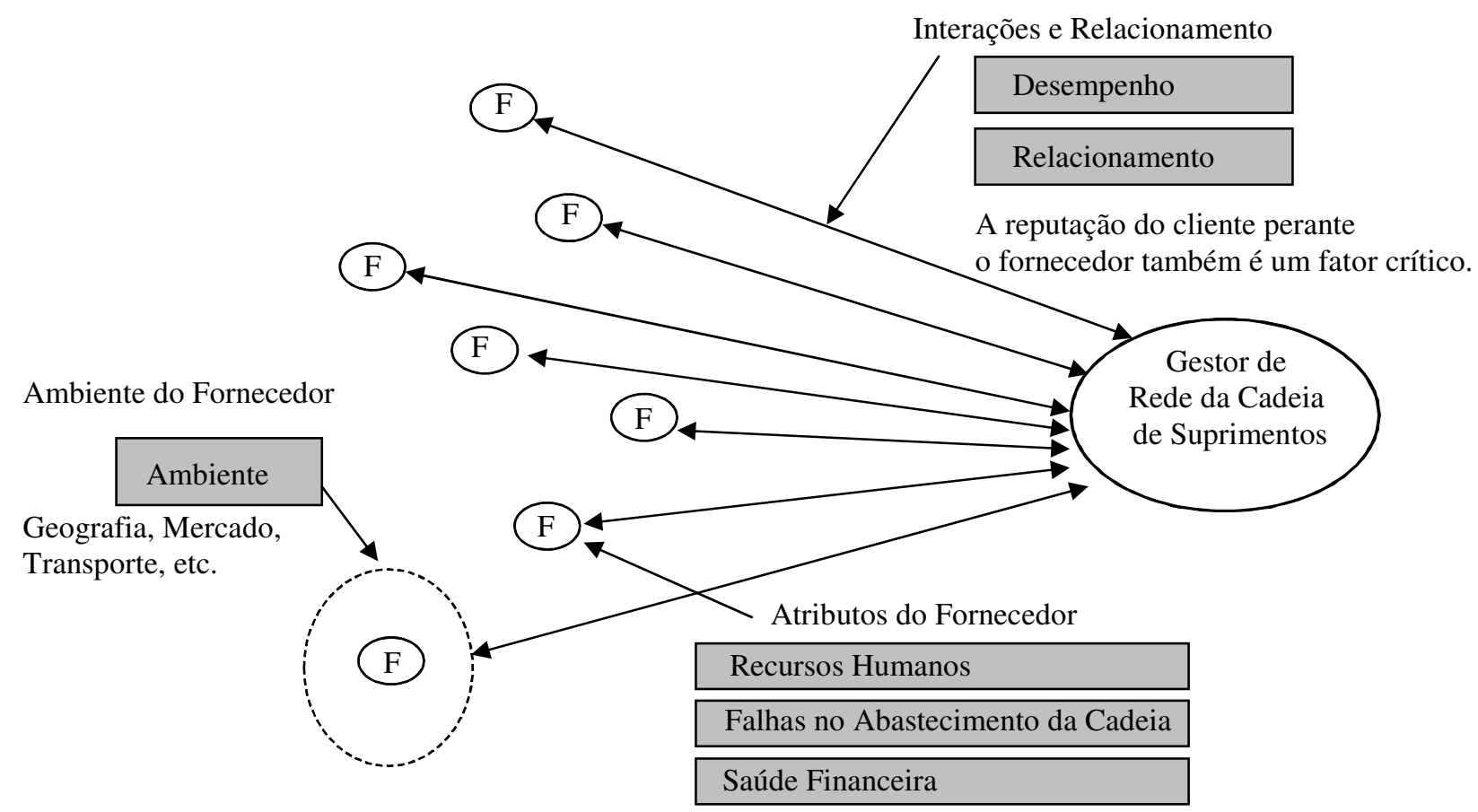

Figura 3: Modelo hipotético para gestão de riscos em cadeias de suprimentos Fonte: Handfield e McCormack (2007)

Este artigo propõe, a partir do modelo hipotético representado na figura 3, que a mensuração do risco de ruptura em cadeias de suprimentos pode ser viabilizada 
por meio de indicadores (Tabela 3) que venham a oportunizar a mensuração do risco de ruptura gerado por cada empresa pertencente à uma ou mais cadeias de suprimentos. Desta forma, tomando-se o risco de ruptura apurado para cada empresa, tornar-se-á possível construir uma rede bayesiana que indique a probabilidade ou o risco de ruptura, reduzindo-se assim os níveis de incerteza associados aos processos de tomada de decisão gerencial.

Das múltiplas fontes potenciais de riscos de ruptura na cadeia de suprimento, outras associações outras também poderiam ser estudadas e investigadas, aumentando, assim, o poder preditivo do modelo, tais como:

1. Quais são os requisitos estruturais necessários para a implementação de modelos de gerenciamento de riscos em cadeias de suprimentos?

2. Como deve ser conduzido ou realizado o planejamento do risco para toda a cadeia de abastecimento?

3. Em que medida o desenvolvimento de novas capacidades de processos afetam os níveis de risco de ruptura nas redes?

4. Como os atributos de performance - responsividade e eficiência - afetam os níveis de risco de rupturas de estoque nos contextos da logística integrada e do gerenciamento da cadeia de suprimentos?

5. O modelo de redes bayesianas poderia ter um maior poder preditivo se utilizado em conjunto com uma estratégia de segmentação de fornecedores, seguindo-se estruturas de governança específicas de parceria e de mercado?

6. Quais os efeitos positivos do gerenciamento de riscos de ruptura sobre os níveis de serviços aos clientes finais de uma cadeia de suprimentos?

Estas e outras questões mobilizam e devem continuar a requerer a atenção de pesquisadores e profissionais da área para o tema. A continuidade de pesquisas e o conseqüente desenvolvimento de novos e melhores modelos validados em pesquisas de campo - em diferentes formatos de amostras e tipos de cadeias de suprimentos - contribuirão para a construção de um corpo de conhecimento teórico sobre o assunto, no contexto da Gestão de Operações. 


\section{CONSIDERAÇÕES FINAIS}

O modelo conceitual apresentado neste trabalho tem como orientação o gerenciamento do risco de rupturas de estoques em cadeias de suprimentos, favorecendo a investigação de uma questão estratégica e bastante sensível às organizações: a oportunidade de que, conhecendo melhor os riscos de ruptura, os níveis de estoque na cadeia possam ser minimizados ao mesmo tempo em que níveis de serviço superiores poderão ser garantidos, em função do maior controle dos stock-outs ou de probabilidades de rupturas de estoques. $\mathrm{O}$ modelo também pode ser útil na determinação dos tipos de relacionamentos a serem desenvolvidos entre empresas dada a maior clareza em relação aos atributos de desempenho que serão monitorados em cada relacionamento. Da mesma forma, poderá auxiliar no mapeamento dos níveis de risco associados a cada fornecedor de uma organização, ou, na perspectiva sistêmica da rede, de todos os fornecedores de uma cadeia de suprimentos.

\section{REFERÊNCIAS}

AZOURY, K. S.; MILLER, B. L. A comparison of the optimal ordering levels of Bayesian and non-bayesian inventory models. Management Science, v.30, n.8, 1984.

BLACKHUST, J.; CRAIGHEAD, C. W.; ELKINS, D.; HANDFIELD, R. B. An empirically derived agenda of critical research issues for managing supply chain disruptions. International Journal of Production Research, v. 43, n.19, p. 40674081, oct., 2005.

CHRISTOPHER, M. Logística e gerenciamento da cadeia de suprimentos. São Paulo: Pioneira, 1997.

ELKINS, D.; HANDFIELD, R. B.; BLACKHURST, J.; CRAIGHEAD, C. W. Ways to guard against disruption. Supply Chain Management Review, jan./feb., 2005. 
FENTON, N.; NEIL, M. Managing risk in the modern world: applications of bayesian networks. London Mathematical Society and the Knowledge Transfer Network for Industrial Mathematics, November, 2007.

FORRESTER, J. Industrial dynamics. Cambridge: MIT press, 1961.

GIUNIPERO, L.; ELTANTAWY, R. Securing the upstream supply chain: a risk management approach. International Journal of Physical Distribution \& Logistics Management, 34, n.9, p.698-713, 2004.

HAMMOND, T. R.; O'BRIEN, C. M.. An application of the Bayesian approach to stock assessment model uncertainty. ICES Journal of Marine Science, v. 58, p. 648656, 2001.

HANDFIELD, R.; McCORMACK, K. Supply chain risk management: minimizing disruptions in global sourcing. Taylor \& Francis, dec. 2007.

LOCKAMY III, L; MCCORMACK, K. Analysing risks in supply networks to facilitate outsourcing decisions. International Journal of Production Research, v. 48, n. 2, p. 593-611, jan. 2010.

MARQUES, R.L.; DUTRA, I. Redes Bayesianas: o que são, para que servem, algoritmos e exemplos de aplicações. Disponível em <http://www.cos.ufrj.br/ ines/courses/cos740/leila/cos740/Bayesianas.pdf>. Acesso em: 24 mar. 2010.

MENTZER et al. Defining supply chain management. Journal of Business Logistics, v. 22, n.2, 2001.

NEIGER, D.; ROTARU, K.; CHURILOV, L. Supply chain risk identification with valuefocused process engineering. Journal of operations management, v. 27, n. 2 , p.154-168, 2009.

FENTON, N.; NEIL, M. Managing risk in the modern world: applications of bayesian networks. London Mathematical Society, 2007.

PAI, R. R.; KALLEPALLI, V. R.; CAUDILL, R. J.; MENGCHU ZHOU. Methods toward supply chain risk analysis. In: IEEE INTERNATIONAL CONFERENCE ON SYSTEMS, MAN AND CYBERNETICS, 5., 2003. Proceedings. Washington, DC, 2003. p. 4560-4565. 
PECK, $\mathrm{H}$. Reconciling supply chain vulnerability, risk and supply chain management. International Journal of Logistics: research and applications, v. 9, n. 2, p. 127-142, 2006.

PHILIPS, J. D.; DAWSON, L.E.Jr. Bayesian statistics in retail Inventory management. Journal of Retailing, v. 44, n. 2, 1968.

RICE, J.; CANIATO, F. Building a Secure and Resilient Supply Chain. Supply Chain Management Review, v. 7, n. 5, p.22-30, 2003.

ZSIDISIN, G.A. Managerial perceptions of supply risk. Journal of Supply Chain Management, v.39, n. 1, 2003.

ZSIDISIN, G. A.; ELLRAM, L.M.;CARTER,J.R.;CAVINATO,J.L. An analysis of supply risk assessment techniques. International Journal of Physical Distribution \& Logistics Management, v. 34, n. 5, 2004.

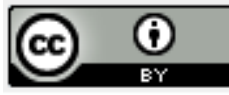

Artigo recebido em 02/06/2009 e aceito para publicação em 04/08/2010. 